\title{
Téoros
}

Revue de recherche en tourisme

\section{Adaptation aux nouvelles technologies dans l'industrie du tourisme}

\section{François Bédard}

Volume 18, numéro 3, automne 1999

Le tourisme dans tous ses états

URI : https://id.erudit.org/iderudit/1071850ar

DOI : https://doi.org/10.7202/1071850ar

Aller au sommaire du numéro

Éditeur(s)

Université du Québec à Montréal

ISSN

0712-8657 (imprimé)

1923-2705 (numérique)

Découvrir la revue

Citer cet article

Bédard, F. (1999). Adaptation aux nouvelles technologies dans l'industrie du tourisme. Téoros, 18(3), 33-39. https://doi.org/10.7202/1071850ar d'utilisation que vous pouvez consulter en ligne.

https://apropos.erudit.org/fr/usagers/politique-dutilisation/ 


\section{Adaptation AuX nOUVElles TECHNOLOGIES DANS L'INDUSTRIE DU TOURISME}

\section{François Bédard}

L'adaptation des entreprises aux nouvelles technologies figure parmi les faits marquants de l'industrie du tourisme en ce début du XXI siècle. Comprenant trois parties, l'article commence par un tour d'horizon des principales problématiques liées à la nouvelle économie. Ce tour d' horizon permet de définir le concept de la nouvelle économie, désignée aussi sous le vocable de commence électronique, d'en mesurer l'importance et, finalement, de cerner les principales problématiques pour les entreprises au regard de ce phénomène, La deuxième partie du texte porte sur tue proposition d'un cadre theorique d'adaptation aux nouvelles technologies dans les services et, en troisième partie, quelques exemples d'initiatives récentes dans le secteur du tourisme servent à illustrer l'opérationalisation de ce cadre théorique.

\section{QUELQUES PROBLÉMATIQUES LIÉES À LA NOUVELLE ÉCONOMIE}

\section{DÉFINITION DES TERMES}

L'innovation et le progrès technologique constituent les deux principales sources motrices de la nouvelle économie que l'on désigne également sous d'autres vocables : l'économie de l'information, l'économieréseau, l'économie numérique, l'économie du savoir - les Américains parlent aussi de la Risk Society - , bref, le commerce électronique.

De quoi s"agit-il au juste? Il est question d'Internet, évidemment ; d'une révolution technologique comprenant les puissants ordinateurs personnels ; d'une planète enveloppée de réseaux de télécommunications. Rapidité, flexibilité, innovation, voilà le credo du commerce électronique.

L'éclatement des frontières se situe au cceur du commerce électronique. Aujourd 'hui, l'entrepreneur n'a plus le choix : il doit penser globalement dès la conception de son entreprise ; il doit s'attarder à scruter le concurrent installé de l'autre côté du globe. Le commerce électronique sur Internet, qui est de plus en plus â la portée de toutes les entreprises, supprime les barrières géographiques et les fuseaux horaires, simplifie et accélère les opérations commerciales et, conséquemment, réduit les coûts : on estime que le coût d'une transaction virtuelle représente le dixiême du coût d'une transaction traditionnelle (Dubé et Paré, 1999: 15).

L'Organisation de coopération et de développement 6 conomiques (OCDE) s' intéresse grandement à la question du commerce électronique. Selon cette organisation, le commerce électronique désigne en général toutes les formes de transactions liées aux activités commerciales, associant tant les particuliers que les organisations, et reposant sur le traitement et la transmission de données numérisées, notamment texte, son et image. Il désigne aussi les effets que l'échange électronique d'information commerciale peut avoir sur les institutions et sur les processus qui facilitent et encadrent les activités commerciales (OCDE, 1997: 13-14).

Bell Canada, la plus grande entreprise de télécommunications au Canada, propose deux définitions du commerce électronique, Dans sa définition large, le commerce électronique représente l'ensemble des activités commerciales réalisées à l'aide des réseaux informatiques tels qu'Internet, y compris l'échange de correspondance électronique ainsi que la promotion ou la vente de produits et de services sur le Web. Dans une définition plus stricte, le commerce électronique désigne la vente de produits ou de services, dont le paiement par carte de crédit et par transfert de fonds (Bell Canada, 1998).

Valdimir Zwass (1998), rédacteur en chef du International Joumal of Electronic Commerce, définit le commerce électronique comme des échanges d'information d'affaires, le maintien de relations d'affaires et l'exécution de transactions commerciales au moyen de réseaux de télécommunications.

\section{IMPORTANCE DU COMMERCE ÉLECTRONIQUE EN CE DÉBUT DE XXI" SIĖCLE}

Bien qu'à l'heure actuelle le commerce électronique soit, pour l'essentiel, utilisé dans les échanges entre les entreprises et entre les organisations, les services de conmerce électronique ciblant les consommateurs individuels se développent rapidement. Internet joue un rôle de catalyseur important dans la diffusion du commerce électronique dans un nombre croissant de 
sphères économiques; il entraîne une harmonisation rapide de l'environnement général dans lequel s'effectuent toutes sortes de transactions électroniques. Il convient donc d'établir un climat de confiance au commerce electronique, pour que l'on puisse retirer le maximum d'avantages économiques et sociaux des nouvelles possibilités qu'il offre.

Le commerce électronique n'en est qu'à ses débuts. Aujourd' hui, il concerne trois types de transactions : la vente de biens matériels, la vente de produits immatériels (ex. : logiciels) ou des services transactionnels (ex. : tourisme, assurances). Il existe également trois types de serveurs Internet commerciaux ; les sites vitrines, les plus nombreux, les sites interactifs, dotés de forums qui permettent de connaitre les attentes des clients, et les vrais sites transactionnels, une minorité pour l'instant, mais en forte croissance. Enfin, le commerce electronique peut aussi bien se limiter à une initialisation en ligne, la commande et le paiement se faisant par des moyens existants (télécopieur, tếléphone et règlement par chèque), ou couvrir l'ensemble d'une transaction (réservation ou commande, puis paiement sécurisé en ligne). De telles conditions rendent toute prévision globale difficile et peu significative en cette période de décollage (Wade et Falcand, 1998 : 148).

Certaines grandes firmes et universités se spécialisent dans des études visant à mesurer l'importance du commerce électronique. À titre d'exemple, au Canada, la Evans Research Corporation estime que le commerce électronique devrait atteindre de 660 à 850 millions de dollars en l'an 2000. Les administrateurs du serveur Yahoo, important moteur de recherche sur Internet, estiment que 60 à 100 nouveaux sites commerciaux à l'échelle mondiale naissent chaque jour sur le Net (Dubé et Paré, 1999: 18). Du côté des firmes américaines, Forrester Research Inc. prévoit que, pour la période de 1999 à 2003 , les echanges Internet entre les entreprises passeront de 43 à 1300 milliards de dollars, alor's que le commerce au détail accélérera deux fois moins vite (de 8 a 108 milliards de dollars). Le secteur des voyages à lui seul enregistrera des revenus de 29,5 milliards (Perreault, 1999). PhoCusWright, firme de recherche américaine, estime que le commerce électronique (ventes sur le Net) dans le secteur du voyage en 1999 totalise 6 milliards de

\begin{tabular}{|l|l|l|}
\hline \multicolumn{3}{|c|}{ TABLEAU 1 } \\
\hline Nivenomie Internet en 1998 \\
\hline & $\begin{array}{l}\text { Estimé des revenus } \\
\text { Internet (Millions de } \\
\text { dollars américains) }\end{array}$ & $\begin{array}{l}\text { Nombre d'emplois } \\
\text { associés à Internet }\end{array}$ \\
\hline Infrastructures Internet & 114982,8 & 372462 \\
\hline Applications & 56277,6 & 230629 \\
\hline Intermédiaires/Développement de marché & 58240,0 & 252473 \\
\hline Commerce sur Internet & 101893,2 & 481990 \\
\hline $\begin{array}{l}\text { Moins ajustements } \\
\text { (croisements et activités interreliées) }\end{array}$ & $(30000,0)$ & $(133755)$ \\
\hline Total & 301393,0 & 1203799 \\
\hline
\end{tabular}

Source ; The Internet Economy Indicators, 20 juillet 1999.

dollars américains. Cinquante-quatre pour cent $(54 \%)$ des ventes sont effectuées par des intermédiaires, et $46 \%$ par les fournisseurs eux-mênes, dont $25 \%$ par les lignes aériennes, $13 \%$ par les hôtels et $8 \%$ par les loueurs de voiture. Cette firme prévoit que les ventes sur le Net dans le secteur du voyage devraient atteindre les 20 milliards de dollars américains d'ici 2001 (ITR, 1999;8).

Le Center for Research in Electronic Commerce - University of Texas a publié à l'été 1999, dans une étude commanditée par Cisco Systems' , des données qui indiquent que le phénomène dans son ensemble aurait dejà atteint une taille très importante. L'économie Internet ${ }^{2}$, appellation qu'ils donnent au commerce électronique dans leur étude, aurait généré plus de 300 milliards de dollars américains en revenus en 1998 et aurait supporté 1,2 million d'emplois. Les chercheurs ont développé une structure à quatre niveaux pour mesurer les revenus et les emplois (tableau 1).

Les niveaux des \& infrastructures w et des * applications * comprennent les investissements dans Internet, les intranets et les extranets. Les niveaux des * intermediaires $w$ et du commerce $w$ ne prennent en compte que les activités liées à Internet.

Comme le soulignent les chercheurs du Center for Research in Electronic Commerce, dans n'importe quelle économie de marché, les règles de la concurrence déterminent les gagnants et les perdants : c'est vrai pour les pays, les entreprises et les personnes. Dans l'économie agraire. la terre et les ressources ont déterminé qui a gagné et qui a perdu. Dans l'économie industrielle, Michael Porter nous a enseigné que la chaîne de valeur — en détenant le plus de contrôle possible - était la base de la concurrence. Dans l'économie Internet, où la connaissance, I'information et le temps sont les monnaies concurrentielles qui prevalent, une nouvelle serie de règles se met en place. Ces nouvelles règles reflètent ce qui est a la base de l'économie Internet : elles s'appuient sur des normes technologiques, elles font la promotion d'une participation du marché grande et étendue géographiquement, tout en encourageant l'anarchie du marché (marché non réglementé). Ces règles sont la source du succès concurrentiel et la route vers la prospérité pour les entreprises et les personnes dans les économies de marche.

\section{PROBLÉMATIQUES POUR LES ENTREPRISES}

Selon l'OCDE (1997), il convient, pour les gouvernements et les entreprises, de développer la confiance au commerce électronique, pour que le plus grand nombre puisse retirer le maximum d'avantages economiques et sociaux des nouvelles possibilités qu'il offre ; à cet effet, tant les gouvernements que les entreprises devront aborder de nombreuses questions. Dans le présent article, nous nous limiterons aux problématiques pour les entreprises.

Pour degager les principales problématiques qui concernent les entreprises, nous traitons successivement du contexte général du commerce électronique du point de vue des entreprises, du commerce elec- 
tronique des biens matériels et des biens immatếriels ou dématérialisés, de la nouvelle distribution et du marketing, en faisant ressortir les differences notables entre la place de marché traditionnelle et le cybermarché.

\section{LE CONTEXTE GÉNÉRAL}

Le Groupe de travail de 1'OCDE s'est également penché sur les problématiques liées au commerce électronique du point de vue des entreprises. Le commerce, rappelle-t-il, repose sur la confiance. Pour que le marché électronique prenne de l'essor, tant auprès des utilisateurs que des entreprises, il faut que les acquéreurs et les vendeurs puissent acoorder aux résultats du commerce électronique un niveau de confiance au moins égal à celui qu'ils accordent aux autres formes traditionnelles de transactions. Chaque participant à une transaction électronique doit être en mesure de $s$ assurer que la transaction et l'environnement de marché dans lequel elle intervient sont légitimes : le vendeur et l'acquéreur sont ceux qu'ils prétendent être ; le vendeur a le droit de vendre l'article en question ; 1 'acquereur dispose des. ressources nécessaires à l'acquisition de l'article ; les mécanismes de transaction et de paiement sont disponibles, légaux et sûrs; l'article vendu correspond à sa description et répond au besoin ; et l'article acquis (qu'il s'agisse d'un bien ou d'un service) peut être livré à l'acquéreur.

De plus, les parties à la transaction s"attendent également à tirer un avantage économique d'un environnement de marché ouvert et concurrentiel, sans distorsions artificielles favorisant certains acteurs par rapport à d'autres. Toutes ces attentes soulèvent un certain nombre de questions fondamentales : où une transaction électronique s' effectue-t-elle réellement, en termes d'obligations contractuelles, d'attribution des responsabilités et de responsabilités fiscales ? Où sont enregistrées et réglementées les entreprises qui pratiquent le commerce électronique et à quels régimes juridiques sont-elles soumises ? Comment peut-on protéger les droits afférents à des formes de propriété matérielles et immatérielles? Que se passe-t-ill lorsqu' une transaction échoue : à qui en incombe la responsabilité ?

Bien que certaines opérations de commerce électronique lancées avec un minimum d'investissements aient été couronnées de succès, particulièrement dans le milieu en pleine éclosion d'Internet, la plupart des entreprises estiment que des investissements substantiels sont aujourd hui nécessaires pour que de telles opérations soient fructueuses. Souvent, la plus grande partic de ces investissements n'est pas constituée par les couts directs d'équipement, mais par les coûts associés à la mise en ceuvre et au suivi de nouveaux systèmes et à l'acquisition de nouvelles compétences technologiques et organisationnelles. Dans les petites et moyennes entreprises ( $\mathrm{PME}$ ). ces coûts peuvent absorber une part des capitaux d'investissement proportionnellement plus importante que dans les grandes societes.

Bien que les technologies de l'information doivent théoriquement permettre d'augmenter les flux d'information accessibles aux acheteurs et aux vendeurs, l'experience des sociétés contactées par le Groupe de travail de l'OCDE montre que le commerce flectronique engendre une conscience accrue de la valeur de l'information en tant que $\alpha$ ressource $*$ et donc une consolidation des réseaux de partenaires, ainsi qu'une réticence à diversifier les partenaires ou à en changer.

Certaines entreprises ont noté, quand il $s^{4}$ agit de choisir un fournisseur dans un environnement électronique, que le prix devient un facteur moins important que la confiance fondée sur une expérience passée avec tel fournisseur et sur les niveaux existants de compatibilité technologique. Enfin, il ne faut pas exclure la possibilité de voir certains détaillants se retourner contre des sociétés qui utilisent le commerce électronique pour accroître leur volume de ventes directes. Ces détaillants * court-circuités * (particulièrement si ce sont de grands groupes) pourraient prendre des mesures compensatoires en limitant l'étalage des produits concurrents dans les magasins, poussant ainsi les fournisseurs à créer des consortiums pour contrecarrer ces mesures de représailles.

\section{LE COMMERCE ÉLECTRONIQQUE DES BIENS MATÉRIELS ET DES BIENS IMMATÉRIELS OU DÉMATÉRIALISÉS}

Le commerce électronique est plus ou moins répandu selon les secteurs. Les médias ont beaucoup insisté sur la vente en ligne de livres, de vins et d'ordinateurs, mais les données disponibles montrent que les ventes de biens immatériels sont les plus importantes : voyages et services de réservation, logiciels, divertissements y compris pour adultes (jeux de hasard, jeux en ligne et musique, notamment) et services financiers. Cela semble logique, les produits immatériels n'ayant par nature pas d'existence physique, le commerce traditionnel ne présente alors aucun avantage par rapport à ceux que procurent les transactions commerciales par voie électronique. Pour ces produits et d'autres biens immatériels - services audio, vidéo et d'information, services immobiliers et services aux entreprises (centres téléphoniques, services de paye et de facturation) - l'impact économique du commerce électronique pourrait être fort et se faire sentir relativement vite. Par la suite, il est probable que cette forme de commerce gagnera toute activité economique susceptible de se prêter à la numérisation, y compris de grands secteurs comme la santé, l'enseignement et de nombreux services publics (Wyckoff, $1997: 6$ ).

\section{LA DISTRIBUTION}

Le commerce électronique réduit radicalement la distance qui sépare les producteurs des consommateur's. Ces derniers peuvent désormais réaliser directement leurs achats sans faire appel aux habituels intermédiaires - détaillants et grossistes - et, dans les cas de biens immatériels, sans distributeur. Même si de nouveaux intermédiaires sont nécessaires (fournisseurs d'accès au réseau, systèmes de paiement électronique, services d'authentification et de certification des transactions, par cxemple), les services qu'ils foumissent exigent beaucoup moins de main-d'ceuvre que les services commerciaux traditionnels et ne demandent pas d'implantation géographique définie. En général, le commerce électronique permet de se rapprocher de certains critères qui définissent une concurrence parfaite: faibles couts de transaction, peu de barriêres à l'entrếe, meilleur accès à l'information pour le consommateur. La relative facilité avec laquelle on peut ouvrir un point de vente sur Internet est un véritable défi pour les producteurs en position dominante qui, auparavant, exerçaient une forte influence sur les réseaux de distribution traditionnels. En effet, les petits producteurs indépendants, où qu'ils soient implantés dans le monde, peuvent doré- 
navant accéder au marché mondial sans trop de difficulté (Wyckoff, 1997: 7).

Les premières études sur le commerce électronique présentent des résultats contradictoires concernant le rôle des intermédiaires. Certains prédisent qu' Internet va amener leur élimination ; d'autres, au contraire, voient leur importance croître. Ces positions contradictoires s'expliquent, selon Bailey et Bakos (Golfinopoulos et Talbot, 1998: 414), par le manque de précisions sur les différentes fonctions effectuées par les intermédiaires. Dans leur étude, les deux auteurs proposent une nouvelle approche qui divise la transaction commerciale en ses différentes composantes et qui analyse les rôles des intermédiaires dans chacune de ces composantes. Selon eux, les fonctions des intermediaires se résument à quatre : agréger la demande des acheteurs ou l'offre des vendeurs (réduction des coûts de transaction, économie d'échelle, réduction de l'asymétrie dans le pouvoir de négociation des vendeurs et des acheteurs); agir comme un agent de confiance (gestion de I'information permettant une transaction limpide de part et d'autre) : faciliter le marché (diffusion de l'information ; production de certains services auxiliaires liés aux paiements, aux formalités administratives) et mailler les besoins des acheteurs et des vendeurs (identification des besoins de chaque partie, connaissance à la fois des produits et des besoins de la clientèle). Ils voient la désintermédiation comme une possibilité et non une certitude. Dans le contexte du commerce électronique, certaines fonctions des intermédiaires pourraient disparaître, mais d'autres vont émerger. En effet, le besoin relatif à la fonction d'agrégation dans le commerce électronique diminuera ; alors que celui qui concerne la relation de confiance augmentera. Quant à la fonction de maillage des besoins - intelligence de marché - celle-ci demeurera.

\section{LE MARKETING}

Les professionnels du marketing font face à un nouveau défi : obtenir du succès dans le cybermarché. Il existe des différences, dont il faut tenir compte, au regard du contenu. du contexte et de l'infrastructure, entre la place du marché traditionnelle et le cybermarché. Tel qu'illustré au tableau 2 , les biens et les services requièrent la présence physique d'un fournisseur

\begin{tabular}{|c|c|c|}
\hline \multicolumn{3}{|c|}{$\begin{array}{l}\text { Principales différences entre la place } \\
\text { de marché traditionnelle et le cybermarché }\end{array}$} \\
\hline Variables & Place du marché traditionnelle & Cybermarché \\
\hline Contenu & $\begin{array}{l}\text { Biens et services qui requièrent la } \\
\text { présence physique d'un fournisseur }\end{array}$ & $\begin{array}{l}\text { Information et services quil ne } \\
\text { requièrent pas la présence } \\
\text { physique d'un fournisseur }\end{array}$ \\
\hline Contexte & Environnement dynamique & Environnements statiques \\
\hline Infrastructure & $\begin{array}{l}\text { Le magasin, les heures d'ouverture, } \\
\text { l'accessibilite au magasin }\end{array}$ & $\begin{array}{l}\text { Adresse, numéro de téléphone, } \\
\text { type de système, oủ conserver } \\
\text { l'information }\end{array}$ \\
\hline
\end{tabular}

Sourcée : Björk et Guss, 1999, p. 67

dans la place du marché traditionnelle, ce qui n' est pas le cas dans le cybermarché La place du marché traditionnelle se situe dans un environnement dynamique, alors que le cybermarché évolue dans des environnements statiques. Finalement, en ce qui a trait à l'infrastructure, la place du marché traditionnelle comporte un lieu physique (le magasin), des heures d'ouverture et des considérations liées â l'accessibilité du lieu. Dans le cybermarché, ce sont les télé́communications et les systèmes de bases de données qui deviennent determinants.

Deux phénomènes, le développement technologique et des consommateurs de plus en plus exigeants, ont crét de nouvelles opportunités de marketing. Nombreuses sont les entreprises qui utilisent Internet, ou plus précisément le Web, pour communiquer avec le consommateur et lui présenter leurs produits ou leurs promotions. II s'agit là essentiellement d'un prolongement du marketing grand public traditionnel, Internet étant une extension des moyens de communication classiques tels que la presse écrite, la radio et la télévision, qui permet en plus l'interactivité. Pour une entreprise, le potentiel d'Internet, du point de vue marketing, peut se résumer aux éléments suivants : accroître sa visibilité et promouvoir ses produits ; représenter un réseau de distribution : communiquer directement avec les clients ; connaître ses clients par differents moyens tels que la visite de forums de discussion. les recherches sur Internet, et les * cookies o (témoins) ; pratiquer un marketing personnalise (one-to-one) ; augmenter la vente croisée : créer des coûts de transfert et fidéliser les clients (Vincent et Ricard, 1998 : 465).
Toutes ces caractéristiques permettent à Internet de contribuer à renforcer les liens entre le client et l'entreprise. En effet, le client a la possibilité de communiquer directement avec une entreprise qui le connaît, l'écoute et lui répond de manière personnalisee. L'entreprise, qui connaît ses préférences et ses habitudes, peut lui faire une offre personnalisée de produits, de services et ou de publicité. Pour atteindre ce résultat, l'entreprise et le client ont dû investir temps et argent dans leur relation, c'est pourquoi une rupture, c'est-à-dire le départ du client vers un concurrent, créerait pour lui des couts de transfert. Ces couts diminuent la propension à devenir infidèle. augmentant du même coup le taux de rétention des clients. Il est cependant important de souligner que tous les consommateurs ne sont pas susceptibles d'adopter de tels comportements. Certaines personnes prennent en effet plaisir à magasiner et pourraient se laisser difficilement séduire par des offres personnalisées. Les personnes qui achètent sur Internet font majoritairement partie des * utilitaristes * (Nantel 1997); ce sont donc des personnes qui ne veulent pas perdre de temps à magasiner et $n^{\prime} y$ prennent pas particulièrement plaisir. Le marketing sur Internet semble davantage s'adapter à ce type de consommateurs (Vincent et Ricard, 1998).

Les consommateurs utilisent actuellement Internet principalement pour obtenir de l'information ou pour s"amuser et très peu pour effectuer des achats. Une étude exploratoire réalisée par Bjôrk et Guss (1999: 54) aupres d'internautes, pour identifier les barrières dans l'utilisation d'Internet comme outil de commerce électronique, a fait ressortir que les consom- 
mateurs démontrent une réticence évidente à utiliser Internet comme outil de commerce ellectronique. Quatre types de barrière ont été identifiés : d'abord l'incertitude perçue par rapport aux systềmes (barrière liée aux expériences personnelles, dimension comportementale) ; puis la nonconfiance à la sécurité d'Internet (barrière liếe aux sentiments personnels, dimension affective); la recherche d'un service personnalise (barrière lié aux sentiments personnels, dimension affective) et, finalement, le scepticisme à l'égard de leur propre comportement (dimension cognitive).

Les fournisseurs peuvent, en adoptant certaines mesures, améliorer la probabilité que les consommateur's deviennent des acheteurs sur Internet, notamment en donnant une rétroaction au client sur ce qui a été acheté ou commandé, en améliorant la sécurité des transactions et en rendant la plus agréable possible l'expérience d'achat sur Internet (Bjorket Guss, 1999). Bref, l'achat en ligne doit se faire de maniere facile et pratique et le prix doit être concurrentiel.

\section{CADRE D'ANALYSE DES STRATÉGIES D'ADAPTATION AUX NOUVELLES TECHNOLOGIES EN TOURISME}

Après ce tour d'horizon des problématiques de la nouvelle économie ou de la societé du savoir, nous nous intéressons maintenant à comprendre comment vont se comporter les intervenants dans l'industrie du tourisme pour s $\mathrm{s}^{\dagger}$ adapter aux defis et aux opportunités découlant de la progression rapide des nouvelles technologies. Nous nous intéressons particulièrement ici aux deux types d'intervenants suivants : les responsables du développement touristique d'une destination (a l'échelle d'un pays, d'une région ou d'une localité) et les PME touristiques.

Nous formulons deux hypothèses :

Pour s'adapter aux défis et aux progrès technologiques, les responsables du développement touristique d'une destination vont avoir tendance à utiliser de plus en plus les possibilites offertes par les nouvelles technologies comme complément a leurs outils traditionnels de marketing. de manière à mieux affronter la vive concurrence dans l'industrie du tourisme, à protéger les parts de marché actuelles et, eventuellement, à les accroître.

Pour s'adapter aux défis et aux progrès technologiques, les PME touristiques vont de plus en plus avoir tendance à créer des partenariats ou à appartenir à des réseaux. à se former et ẩ se tenir à jour en matière de nouvelles technologies et à se doter d'outils technologiques répondant à leurs besoins (tant pour la gestion interne de l'entreprise que pour la commercialisation et la distribution de leurs produits et services).

\section{CONCEPTUALISATION DES HYPOTHËSES}

Le concept étudié est celui du comportement stratégique des responsables du développement touristique d'une destination et des PME touristiques, au regard des nouvelles technologies, dans le contexte de la nouvelle économie et de la sociétét du savoir.

\section{IDENTIFICATION DES DIMENSIONS DU CONCEPT}

Le concept du comportement stratégique des entreprises touristiques s'articule autour de trois dimensions (figure 1) dans le triangle d'adaptation aux nouvelles technologies dans les services: $*$ la formation, l'acquisition et l'utilisation *. La dimension \& formation aux nouvelles technologies se se situe au sommet du triangle pour bien demontrer son importance stratégique dans la société du savoir. Les dimensions * acquisition ou accès aux nouvelles technologies * et «utilisation ou usage des nouvelles technologies $*$ se situent à la base du triangle. Les segments reliant les différentes dimensions indiquent les interrelations entre elles. Le triangle est encadre par quatre synonymes : a partenariat, alliance, regroupement, consortium $s$ pour illustrer les differentes formes de maillage ou de réseautage dans lesquelles évoluent de plus en plus d'entreprises à l'ère du commerce électronique.
FIGURE 1

Le triangle d'adaptation aux nouvelles technologies dans les services

\section{PARTENARIAT}

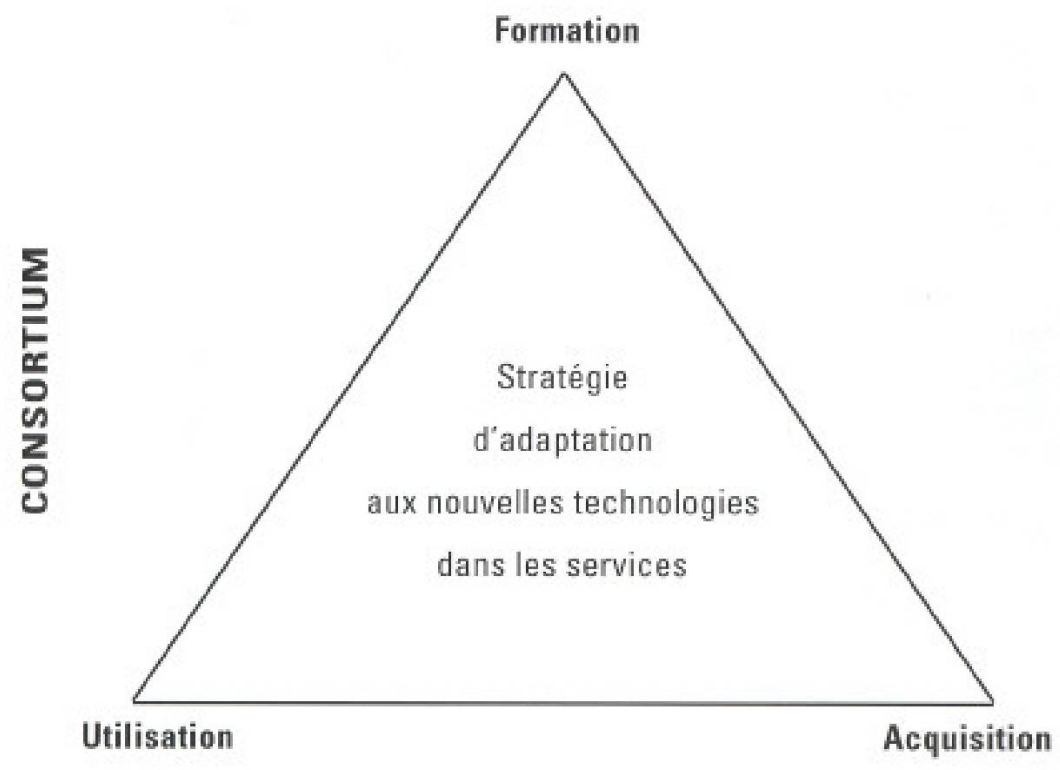

ALLIANCE 


\section{CARACTÉRISTIQUUES DE LA DIMENSION FORMATION AUX NOUVELLES TECHNOLOGIES}

Le mot formation est utilisé ici dans un sens large et comprend également les activités de sensibilisation, de veille et de diffusion des connaissances liés aux nouvelles technologies.

Dans un monde où les changements induits par les nouvelles technologies sont constants, les activités d'acquisition de connaissances et de formation de la main-d'ceuvre occupent une place stratégique dans les entreprises qui désirent demeurer concurrentielles. Outre l'embauche de nouveaux employés qualifiés et formés dans des institutions de formation, les entreprises doivent également former le personnel en place aux nouvelles technologies. Êléments caractéristiques de la société du savoir, la formation et les activités connexes (sensibilisation, diffusion, veille technologique) doivent s'inscrire dans un processus continu (autrement dit, dans les activités régulières des entreprises).

\section{CARACTÉRISTIQUES DE LA DIMENSION ACQUISITION DES NOUVELLES TECHNOLOGIES}

Une autre facette importante du triangle d'adaptation a trait à l'acquisition des nouvelles technologies. Plusieurs questions se posent à cet égard : Quelle part de son budget l'entreprise doit-elle consacrer à l'acquisition des nouvelles technologies? Quelles technologies répondent vraiment aux besoins de l'entreprise ? Qui sont les fournisseurs? Comment et où trouver les produits et les solutions technologiques représentant les meilleurs rapports qualitél prix ? A quelle fréquence faut-il effectuer des mises à jour ou le renouvellement du matériel informatique ? Faut-il acheter des produits technologiques génériques ou en faire développer sur mesure? Quels sont les avantages à faire partie d'un regroupement ou d'un réseau qui fournit les solutions technologiques? Voilà quelques exemples de questions qui font ressortir les problématiques liées à l'acquisition des nouvelles technologies.

\section{CARACTÉRISTIQUES DE LA DIMENSION UTILISATION DES NOUVELLES TECHNOLOGIES}

La dimension utilisation refere aux usages que les entreprises font des nouvelles technologies. On distingue deux principaux usages auxquels correspondent des technologies spécifiques, soit les technologies liées aux opérations internes de l'entreprise (gestion, contrôle) et celles liées aux activités de marketing (distribution, promotion, publicité, fidélisation de la clientèle). Les technologies liées à Internet sont considérées aujourd'hui comme les plus stratégiques dans le contexte de la nouvelle économie. Fait à souligner, les produits du tourisme et du voyage figurent présentement au premier rang des achats effectues par les consommateurs sur Internet.

\section{CARACTÉRISTIQUES DE L'ENCADREMENT DU TRIANGLE D'ADAPTATION AUX NOUVELLES TECHNOLOGIES}

Avec l'avènement de la nouvelle économie, on a vu croître de façon significative le nombre de partenariats, de regroupements, d'alliances et de consortiums. Ces mots, qui représentent autant de formes d'association, encadrent le triangle d'adaptation aux nouvelles technologies pour refléter la tendance que l'on observe depuis quelques années.

\section{OBSERVATIONS ET ANALYSE}

Dans la partie observations et analyse, nous illustrons le triangle d'adaptation aux nouvelles technologies par trois exemples. Le premier porte sur le Centre d'affaires électronique inauguré à la fin de 1999 par Tourisme Québec et Bell Canada: le deuxième, sur l'Institut canadien dı toırisme et du commerce electronique / Canadian Institute of Tourism and Electronic Commerce (CITEC) crée en 1998 et le troisième, sur Tourisme Montréal (autrefois l'Office des congrès et du tourisme du Grand Montréal). Ce dernier cas est présenté dans un texte distinct sous la plume de Pierre Bellerose, directeur, du Développement des affaires et des relations publiques.

Le Centre d'affaires electronique (C.A.E.), connu sous le nom de Bonjour-Québec. com, se situe principalement dans la dimension utilisation des nouvelles technologies telle que définie dans le cadre de référence proposé. En effet, le C.A.E. vise à combler plusieurs besoins, notamment le raffinement des méthodes de promotion et de commercialisation, l'amélioration de la diffusion d'information sur les produits touristiques du Québec, la cueillette de données sur les différentes clientèles et la mise en commun de toutes ces connaissances (Hansen, 1999a : 2). Le C.A.E. recoupe également la dimension acquisition de nouvelles technologies, car il constitue une solution technologique offerte aux entreprises touristiques québécoises. Cette solution comprend plusieurs volets, notamment des services de réservation et des fonctionnalités transactionnelles (Hansen, 1999b : 3). Ainsi, les entreprises participantes font une économie quand elles décident elles-mêmes des technologies qu'elles devront acquérir pour participer à l"économic du savoir et du commerce

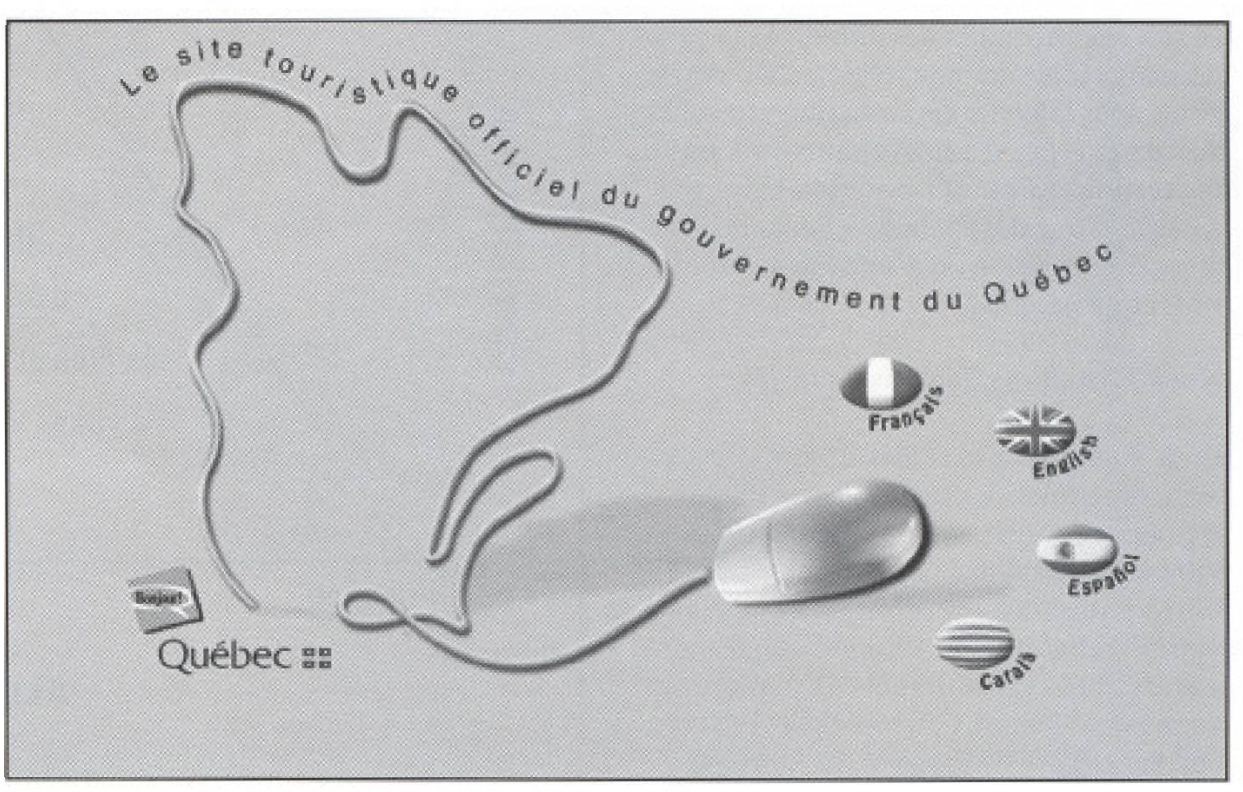


électronique. L'approche de type coopératif permet aux entreprises de la destination Québec d'accéder aux technologies de pointe à un moindre coût, étant donné les économies d'échelle réalisées grâce au grand nombre de participants au système et à la contribution financière de Tourisme Québec.

L'Institut canadien du tourisme et du commerce électronique (CITEC) a été créé en 1998 dans le but d'aider les entreprises touristiques canadiennes, et tout particulièrement les PME, à prendre le virage du commerce électronique et ainsi devenir plus compétitives. Par să mission, CITEC se situe principalement dans la dimension formation du triangle d'adaptation aux nouvelles technologies. CITEC vise à encourager et à faciliter l'inclusion des technologies de l'information, et tout particulièrement du commerce électronique, dans la mise en marché des PME de l'industrie touristique (CITEC 1999). Parmi ses moyens d'intervention figurent la veille technologique, l'information et la formation. Se situant à la tête du triangle d'adaptation, la dimension formation nourrit les deux autres, soit les dimensions acquisition et utilisation des nouvelles technologies. Cette réalité est présente dans les moyens d'intervention de CITEC qui comprennent également la promotion, le développement et la représentation en matière de commerce électronique.

Finalement, tout comme le C.A.E., Tourisme Montréal agit principalement dans la dimension utilisation du triangle d'adaptation. Pour connaitre ses initiatives au regard des nouvelles technologies depuis 1995, nous référons le lecteur à l'article de Pierre Bellerose ayant comme titre « Tourisme Montréal : une démarche à 1"avant-garde des technologies $*$ dans ce numéro de Téoros.

\section{CONCLUSION}

Comme tout autre secteur dans les services, l'industrie du tourisme n'échappe pas à la nécessité de s'adapter à la nouvelle économie ou à l'essor du commerce ellectronique. Les experts prédisent une croissance importante - quasi exponentielle - des ventes effectuées en ligne au cours des trois à cinq prochaines années. Le triangle d'adaptation aux nouvelles technologies dans les services a été proposé comme cadre théorique pour servir de cadre de ré- férence aux gestionnaires. Certaines initiatives ont vu le jour récemment dans le but d'aider l'industrie touristique à effectuer le virage technologique. La création récente du Centre d'affaires électronique Bonjour-Québec.com et de CITEC, ainsi que la démarche entreprise depuis 1995 par Tourisme Montréal, sont autant d'exemples qui témoignent des efforts de l'industrie pour trouver une réponse collective qui permettra de relever avec succès les défis posés par les nouvelles technologies.

François Bédard est professeur au Département d'études urbaines et touristiques, de l' Université du Québec à Montréal. Ses principaux intérêts dans l'enseignement et la recherche portent sur les nouvelles technologies et le tourisme. Il deposera au débu de l'année 2000 une thèse de doctorat sur ce theme à l' Universite de Paris I Pantheon-Sorbonne. A titre de coordonnateur adjoint du Centre international de formation et de recherche en tourisme de I'UQAM (CIFORT), II préside le Chapitre pour les Aneriques de la International Federation on Information Technology and Tourism.

\section{NOTES}

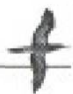

1 Cisco Systems était en 1999, à l'échelle mondiale, le plus grand site de commerce électronique sur Internet, avee des ventes de produits de 28 millions de dollars par jour (donnée publiée par l'entreprise).

2 L'économie Internet est formée de compagnies qui génèrent leurs revenus, en tout ou en partie, à partir d'Internet ou de produits ou services liés à Internet. Ces entreprises sont les joueurs dans les infrastructures Internet et les applications Internet tels que Cisco, Dell, IBM, HP. Oracle, Microsoft et Sun, dont les produits et les services rendent possible l'utilisation d'Internet pour le commerce electronique. Source: The Internet Economy Indicators (juillet 1999).

\section{BIBLIOGRAPHIE}

Bell Canada (1998), Le guide Bell, $\mathrm{n}^{\circ} 2$, Montréal.

Bjôrk, Peter, et Thomas Guss (1999), * The Internet as a Marketspace - The Perception of the Consumers so Dimitrios Buhalis et Walter Schertler, Information and Communication Technologies in Tourism 1999, Vienna, Springer, p. 54-65

Dube, Line, et Guy Paré (1999), * Les technologies de l'information et l'organisation à l'ère du virtuel *, Revue Gestion, wol. $24, \mathrm{n}^{\mathrm{n}} 2, \mathrm{p} .14-22$.

Golfinopoulos, George, et Jean Talbot (1998),
* Commercial Intermediaries on the Internet: Towards a New Perspective *, Gilles SaintAmant et Mokhtar Amami. Commerce electronique - III Colloque Imiernational de Management des Réseaux d'Entreprises, Montréal, Université du Québec à Montréal.

Hansen, Nathallic (1999a), Survol des services du C.A.E. vol. 1, n' 1, Montréal, p. 2.

Hansen, Nathalie (1999b), Tout, tout, tout sur le Centre d'affaires électronique, vol. $1, \mathrm{n}^{\circ} 2$. Montréal, p. 3.

Harvey, Pierre-Léonard (1995), Cyberespace et communautigue, Sainte-Foy, Les Presses de l'Université Laval, 239 p.

Institut canadicn du tourisme et du commerce électronique (1999), Commence electronique l'industrie torristique canadienne à la conquête da nouveau marché mondial (dépliant), Shawinigan (Québec).

Interactive Travel Report (ITR) (1999), PhoCusWright's findings, 13 mai, p. 8 .

Organisation de coopération et de développement économiques (OCDE) (1997), Le commeree électronique : opportunités et défis pour les golwernements, Paris : Rapport d'un groupe d'experts du secteur privé sur le commerce électronique présidé par John Sacher, p. 91.

Perreault, Mathieu (1999), \& Internet séduit les comptables $\%$, La Presse, 9 juillet, p. C: 1 .

The Internet Economy Indicators (1999), The Internet Economy Indicators - Indicators Report, Adresse sur le Web: www.internetindicators.com/, Accès 20 juillet 1999 .

Vincent, Karine, et Line Ricard (1998), * Internet au service du marketing \$, Gilles Saint-Amant et Mokhtar Amami. Commere électronigue - $I / T$ Colloque International de Management des Réseaux d'Entreprises, Montréal, Université du Québec à Montréal.

Wade, Philip, et Didier Falcand (1998), Cyberplanète, Paris, Éditions Autrement - Collection Mutations, $\mathrm{n}^{\circ} 176$, p. 349.

Wycoff, Andrew (1997) \& Imaginer l' impact du cybermarché $\%$, L'Observateur de L'OCDE, vol. 208, p. $5-8$.

Zwass, Valdimit (1998), « Electronic Commerce: Structures and Issues $*$, Gilles SaintAmant et Mokhtar Amami, Commerce electronique = I/ Colloque International de Management des Réseaux d'Entreprises, Montréal, Université du Québec à Montréal. 